\title{
Prolonging Gastrointestinal-Stromal-Tumor-free life, an optimal suggestion of imatinib intervention ahead of operation
}

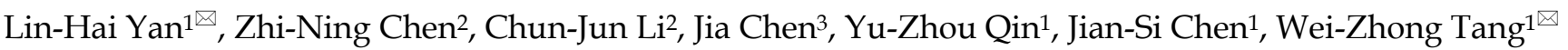 \\ 1. Department of Gastrointestinal Surgery, Affiliated Tumor Hospital of Guangxi Medical University, Nanning 530021, Guangxi Zhuang Autonomous Region, \\ China \\ 2. Department of Pathology, Affiliated Tumor Hospital of Guangxi Medical University, Nanning 530021, Guangxi Zhuang Autonomous Region, China \\ 3. Department of Medical Image Center, Affiliated Tumor Hospital of Guangxi Medical University, Nanning 530021, Guangxi Zhuang Autonomous Region, \\ China \\ $\triangle$ Corresponding authors: Dr. Lin-Hai Yan and Wei-Zhong Tang, Department of Gastrointestinal Surgery, Affiliated Tumor Hospital of Guangxi Medical \\ University, No. 71, Hedi Road, Nanning 530021, Guangxi Zhuang Autonomous Region, China. Tel: +86-771-5344230; E-mail: lhyan@gxmu.edu.cn (L.H.Y.) and \\ 2354531686@qq.com (W.Z.T.) \\ (c) Ivyspring International Publisher. This is an open access article distributed under the terms of the Creative Commons Attribution (CC BY-NC) license \\ (https://creativecommons.org/licenses/by-nc/4.0/). See http://ivyspring.com/terms for full terms and conditions.
}

Received: 2018.01.31; Accepted: 2018.04.14; Published: 2018.10.05

\begin{abstract}
Background: Imatinib has been regarded as the first successful synthetic small molecule targeting at blocking tyrosine kinase. Its high efficacy stabilized disease in above $80 \%$ of chronic myeloid leukemia (CML) patients over 10 years survival. Due to the similar canceration of gastrointestinal stromal tumor (GIST) as to CML, imatinib has been approved to be used as first-line treatment.

Study design: Our retrospective study was proposed to enroll 191 GIST patients with larger tumor size $(\geq 8 \mathrm{~cm})$ who preoperative accepted imatinib from those with direct operation. Analysis included demographics, cancer specific survival and relationship of their risk factors.

Results: Male patients and gastrointestinal (GI) tract location took higher proportion in total cases, detection of KIT mutant took $89.7 \%$ among all traceable genetic testing. Patients with preoperative imatinib can achieve higher cancer specific survival (CSS) after both in 1 year and 3 years duration than their counterpart. Tumor size above its threshold of $8 \mathrm{~cm}$ would be a hazardous factor for poor prognosis.

Conclusion: In conclusion, as for regressing tumor progression and creating operative chance, preoperative imatinib should be considered for the patients with high risk, although the precise duration of this intervention needs further validation.
\end{abstract}

Key words: Gastrointestinal stromal tumor, Neoadjuvant imatinib, Surgical resection, Cancer-specific survival

\section{Introduction}

Imatinib, a small molecule targeting at blocking tyrosine kinase, is the first approved tyrosine kinase inhibitor aiming at effectively inhibited progression of chronic myelogenous leukemia (CML). Genetic change of CML has been confirmed which located at chromosome fusion of 9 and 22, resulting in uncontrollable activation of tyrosine kinase [1, 2]. When Philadelphia chromosome was created after gene fusion of $B C R$ and $A B L$, the increased tyrosine kinase activity happened to match this highly specific agent [3]. The mechanism of action of imatinib is decreasing the phosphorylation of tyrosine kinase by blocking BCR-ABL ligand, though imatinib not only targets $\mathrm{BCR}-\mathrm{ABL}$, but also some of the growth factor receptors [4]. The single initiation of oncogene in CML ensures high selectivity of imatinib, successfully achieving five year survival in 95\% CML patients, and more than $80 \%$ of CML patients can stably alive longer than 10 years $[1,2,5]$.

Few years later, instruction of imatinib was 
revised with additional administration guide of gastrointestinal stromal tumor (GIST). GIST remains a distinct gastrointestinal mesenchymal tumor in GI tract [6]. Misdiagnosis and incomplete pathological classification used to result in low incidence of GIST, but escalating incidence along with the prevalent precision of its origins and morphology distinguished GIST from gastric leiomyoblastoma [6]. Tracking to molecular change of GIST, wherein the KIT gene is abnormally activated or mutated, uncontrollable phosphorylation of tail of its receptors domain constantly stimulates survival pathways $[7,8]$. This unique characteristics of GIST cells also respond to imatinib treatment. Indeed this successful medication creates the era of imatinib in stabilizing GIST progression and recurrence. We shall resist to satisfaction of imatinib since the non-KIT mutation of GIST appears to less effective remission of tumor progression [9]. Although there are other tyrosine kinase inhibitors on the selective table, increasing dosage of imatinib still can achieve a reasonable respond rate to GIST [5]. Due to the strong efficacy to GIST which obtains low sensitivity to systemic chemotherapy, imatinib is still firmly itself in GIST chemotherapy.

\section{Materials and methods}

\section{Patients}

One hundred and ninety one patients with pathological diagnosis of GIST were retrospectively enrolled from January 2012 to December 2014 from 2 major medical centers in Guangxi. All patients provided written informed consent. The enrollment criteria of this study are 1 ). aged 18 - 82 years; 2 ). The location of primary GISTs was in the stomach, intestines, or enterocoelia; 3). Imaging manifestations supported the tumor diameter was required to be larger than $3 \mathrm{~cm}$ and metastasis on liver, lung or peritoneal; 4). All GISTs were first diagnosed without any medical intervention, including radiotherapy, surgery, and chemotherapy; 5). Standard treatment of oral imatinib $400 \mathrm{mg}$ daily which was followed the guidelines indication [10]. The protocol was designed in accordance with the Declaration of Helsinki. The study was approved by the ethics committee of the Guangxi medical university.

\section{Study design and treatment}

Treatment duration of enrolled cases should be at least three months of standard imatinib after pathologically diagnosis of primary GISTs. We divided and renamed all cases into two types (effective type and invalid type) based on the disease response. Effective type of GIST could be operated at the 4 th or 8 th week after neoadjuvant imatinib, and treatment was withdrawn at 4 weeks before surgery. Invalid type could continue medication with imatinib till the 12th week. Timing of surgical resection was determined after evaluating imaging information through computed tomography (CT), Tumor computed tomngraphy angiography (DSCTA) and 3-dimension (3D) reconstruction. Surgically removing the GISTs was performed within 4-6 weeks of final, imatinib treatment was finally discontinued at least 4 weeks before operation. Surgical procedures and reconstruction methods were not expatiated. The intention of surgery would be R0 resection (macroscopically and histologically negative margin). When $\mathrm{R} 0$ resection can not be achieved, in order to delay end point, compromised R1 (histologically positive margin or tumor rupture) or R2 (macroscopically residual tumor) resection were allowed. Imatinib treatment should be post-operatively restarted for at least 1 to 2 years duration.

\section{Data collection}

Enrollment population of 191 patients with demographic details were illustrated in Supplemental material (Table S1). The primary end point was cancer-specific survival (CSS) rate, and the secondary end point was $\mathrm{R} 0$ resection rate. Tumor response was evaluated in accordance with the RECIST, v1.1, every 2 months after initiation of imatinib. Confirmation of $\mathrm{CR}$ (complete remission) or PR (partial remission) was excluded. The tumor responses evaluations were performed by surgeon and radiologists. In the first year and following 2 years, post-operation assessment of recurrence and metastasis was conducted through CT imagines of whole body every 3 months and 6 months respectively. Whole-coding regions of KIT and PDGFRA cDNAs or selected regions of KIT genomic DNA (exons 9, 11, 13, 17) and PDGFRA genomic DNA (exons 12, 14, 18) were sequenced by Genetic testing laboratory of Beijing Tumor Hospital (Beijing, China). Pathological and immunohistochemistrical analysis were carried out by two experienced pathologists. Demographic information was collected as age at diagnosis, gender, Race (Han or Zhuang), primary site of tumor (stomach, intestines, and enterocoelia), tumor size $(<8$ or $\geq 8)$, directly surgical resection (yes or no), and survival months. Cancer-specific survival (CSS) was defined as the period from time of diagnosis to time of death.

\section{Statistical analysis}

The primary endpoint and the secondary endpoint were calculated by Kaplan-Meier method 
with log-rank test. Chi-square test was utilized to compare the differences in clinical and demographic features between groups. The associations between demographic factors with receipt of surgical resection were evaluated using Logistic regression analysis. Multivariable survival analyses of CSS was conducted using the Cox proportional hazards model. $P<0.05$ was considered as statistically significant. All statistical analyses were performed using Stata Statistics 12.0 (StataCorp LLC, Texas, USA) or Data were analyzed using SPSS 19.0 (IBM, USA).

\section{Results}

\section{Patient characteristics}

Demographic distribution of 191 GIST patients were described in Table S1 (Supplemental material). All of GIST patients accepted R0 or R1 resection. Forty seven patients $(24.6 \%)$ received neoadjuvant imatinib before surgical resection while 144 (75.4\%) of whom accepted direct resection alone. Male patients took a higher proportion of morbidity $(60.73 \%, 116 / 191)$ than female patients $(39.27 \%, 75 / 191)$. The characteristics of diseases such as tumor diameter larger than $8 \mathrm{~cm}(60.73 \%)$, location of GI tract $(73.30 \%)$, and KIT mutation $(45.55 \%)$ were appeared among the reviewed cases. The multidrug resistance-associated pathological features included VEGF and EGFR expression, and GIST cell mitotic count among patients carrying different mutation were showed in Figure 1A. A waterfall plot individually represented the downsizing efficacy of imatinib on tumor volume before surgery in Figure 1B. The median reduction rate was $29.8 \%$ (1.5-52.2\%), and neoadjuvant imatinib brought favorable shrinkage of tumor even in 5 patients with wild-type GIST.

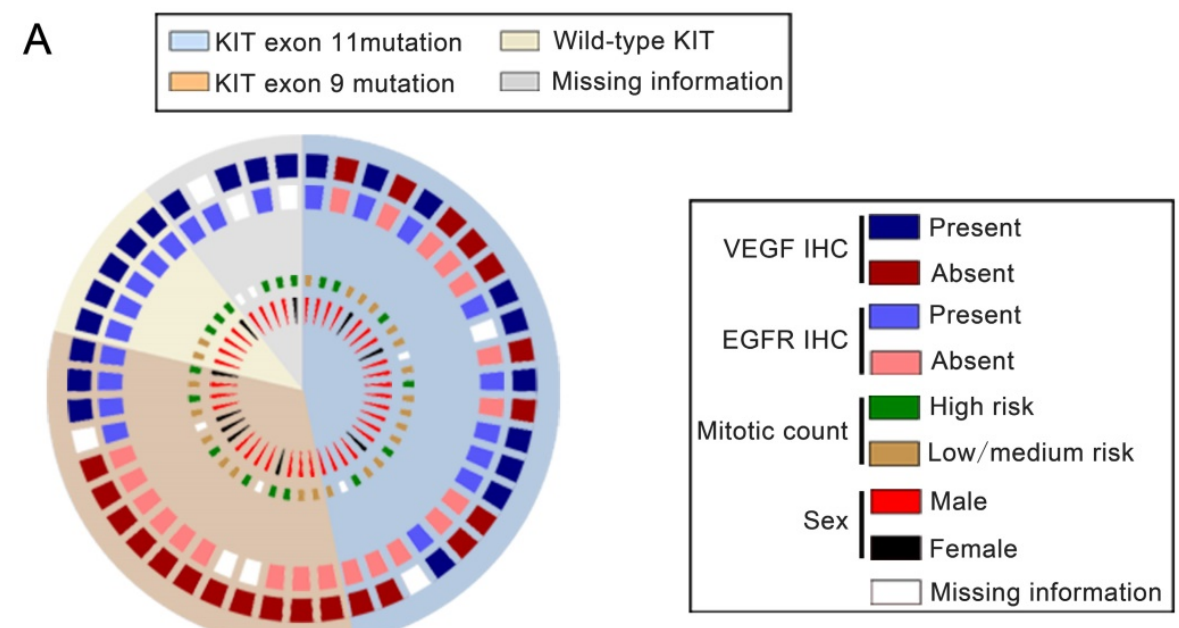

B

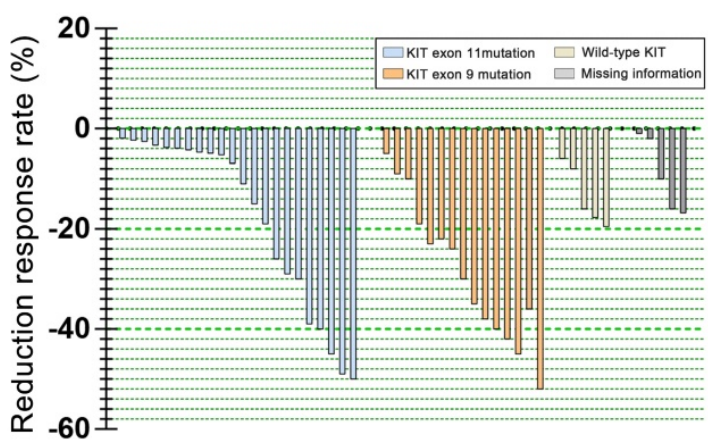

C

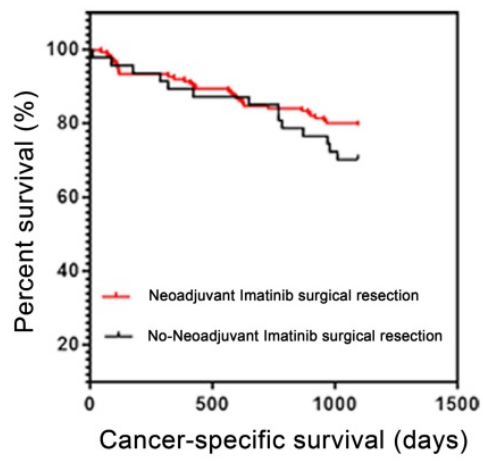

Figure 1. (A) Immunohistochemical analysis (IHC) and protein characteristics of tumors from 47 neoadjuvant imatinib patients With KIT/PDGFRA Wild-Type GIST. The information of pie chart is consist of four concentric circles which was separated by four circular sector. Two of the outer circle represented whether the IHC analysis provided evidence for positive detection of EGFR or VEGFR. The inner circle with squares represented the status of mitotic level. The most inner wedge-shaped circle showed the gender distribution among 47 patients who accepted preoperative neoadjuvant imatinib. The four circles were separated by four sectors, which were showed as different color as background. Each sector illustrated a group of gene status. EGFR, epidermal growth factor receptor; VEGFR, vascular endothelial growth factor. (B) Waterfall plot of tumor shrinkage after neoadjuvant imatinib. The bar chart with four different color demonstrated the reduction of respond rate of each patient who carried individual gene mutation. (C) Survival curves with log-rank test of cancer-specific survival (CSS). The survival rate of patients who accepted neoadjuvant imatinib from onset of medication days in red, while the black line chart showed the tendency of survival percent of patients who accepted direct operation under the same time duration in black. 


\section{Survival comparison}

Mortality occurred in 47 (24.6\% of 191) patients at the end of follow-up. GIST recurrence and metastasis lead to descent among 45 (23.5\% of 191) patients, and the other 2 (1.1\% of 191) decease was caused by postoperative infection. The 1-year CSS rates was $95.53 \%$ in neoadjuvant imatinib group and $89.17 \%$ in no neoadjuvant imatinib group respectively, and the 3 -year CSS rates were $81.25 \%$ in neoadjuvant imatinib group and $69.17 \%$ in no-neoadjuvant imatinib group respectively (Figure 1C). Change in other $\mathrm{R} 0$ resection rates are shown in Table 2. Among all patients, $87.23 \%$ of patients preoperatively accepted imatinib whereas $79.86 \%$ of patients accepted directly process $\mathrm{R} 0$ resection alone. $\mathrm{R} 0$ resection rate was higher in neoadjuvant groups than their counterpart in all 3 observed sites, the statistical difference among $\mathrm{R} 0$ rate of each sites were not statistic significant (Table 1).

A

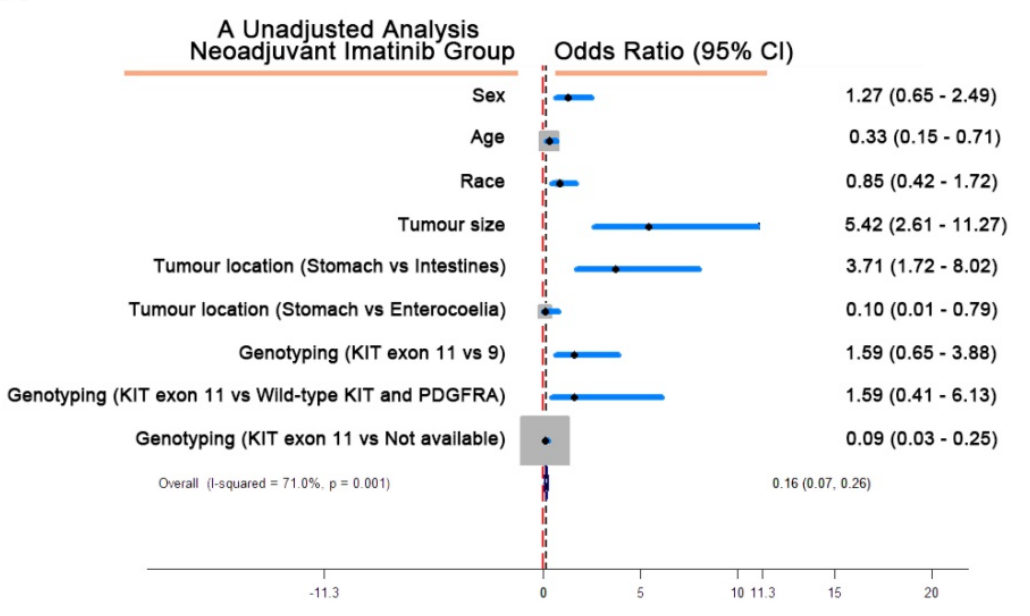

B

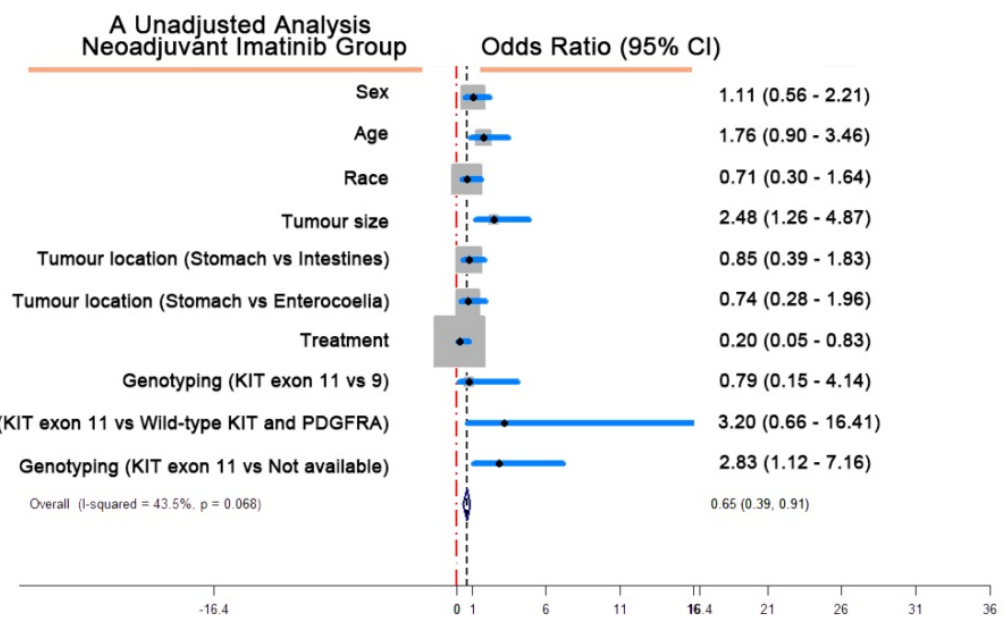

Figure 2. (A) Factors associated with receipt of neoadjuvant imatinib of the primary tumor. The blue scope represented the $95 \mathrm{Cl}$ range of each factor. $\mathrm{Cl}$, confidence interval. (B) Multivariate analysis of cancer-specific survival (CSS). The blue scope represented the $95 \mathrm{Cl}$ range of each factor. $\mathrm{Cl}$, confidence interval.
Table 1. The secondary end point analysis (Completeness of surgery, R0 resection rate)

\begin{tabular}{llll}
\hline & $\begin{array}{l}\text { Neoadjuvant } \\
\text { Imatinib }\end{array}$ & $\begin{array}{l}\text { No-Neoadjuvant } \\
\text { Imatinib }\end{array}$ & $P$ \\
\hline Tumor Location & & & \\
Stomach & $17 / 19(0.89)$ & $46 / 52(0.88)$ & 0.7005 \\
Intestines & $15 / 16(0.94)$ & $44 / 53(0.83)$ & 0.5472 \\
Enterocoelia & $9 / 12(0.75)$ & $25 / 39(0.64)$ & $<0.001$ \\
\hline
\end{tabular}

Patients with GIST (all types: stomach, intestines and enterocoelia) treated by resection followed by neoadjuvant imatinib or no intervention. Data are shown as $\mathrm{R} 0$ resection number/ headcount (R0 resection rate). $p$ values are for Neoadjuvant imatinib vs. No-neoadjuvant imatinib.

\section{Factors in estimation of GIST}

In order to better understand the effect of neoadjuvant imatinib in GIST, we analyzed the clinicopathological factors associated with this intervention (Table 2). Tumor size with the threshold of $8 \mathrm{~cm}$ would be a remarkable indicator to estimate whether neoadjuvant treatment should be considered.

Multivariate Cox regression analysis revealed that medication with neoadjuvant imatinib associated with better CSS (Table 3), the unadjusted analysis demonstrated that over $8 \mathrm{~cm}$ of GIST indicated hazardous situation from which imatinib treatment would gain CSS benefits (Figure 2).

\section{Discussion}

GIST has a short history since it was separated from ambiguous gastric cancer categories [6]. Epidemiological study of GIST illustrated the people aged older than 50 years took about $80 \%$ of total incidence [11, 12]. However in our region, an the advanced GIST lean to affect people younger than 50s. This tendency to young people was also showed in a similar study [13]. The younger trend phenomenon crack down patients mind and life quality. Major treatment relied on complete resection of macroscopic GIST, which would be achieved with long duration of tumor-free survival [14, 15]. Surgery still can not guarantee eradication of microscopic lesion since the origins of GIST would easily invade circulation system. Those microscopic lesion would be a 
latent crisis to trigger recurrence or distant metastasis. Therefore postoperative imatinib treatment regardless duration time could stabilize disease progression. Recommendation of duration of imatinib therapy long to 3 years basically reached agreement $[16,17]$. Should preoperative medication further enhance its efficacy and increase the survival time? Post-operative plasma concentration of imatinib fluctuated among gastrectomy and no surgery patients [18]. Low level of imatinib reduced the binding effect to targeted kinase, even triggered the second mutation. Full function of stomach provides a suitable acidic environment for absorption of imatinib, it is essential for verifying preoperative imatinib might shorten the duration of medication when reached a similar endpoint. Emerging evidence showed newfound subtype of GIST possessed high malignancy and fast growth ability [19]. Therefore, the escalating tumor size illustrated gradual increase of hazardous risk associating with rise of relapsed rate $[10,20]$. Accordance with our results, confirmed pathological diagnosis in male patients were higher and patients aged 50 years older would bear higher risk. Neoadjuvant imatinib is strongly recommended since hazardous risk can be reduced to about 20\% compared with direct resection. Regression of risk level not only increased the completion rate, but also benefited in improving prognosis. Suggested dose of $400 \mathrm{mg}$ of imatinib could delay the recurrence of GIST [16].

Table 2. Factors associated with receipt of neoadjuvant imatinib of the primary tumor

\begin{tabular}{|c|c|c|c|c|c|c|}
\hline & \multicolumn{3}{|c|}{ Univariate model } & \multicolumn{3}{|c|}{ Multivariate model } \\
\hline & OR & $(95 \% \mathrm{CI})$ & $P$ & OR & $(95 \% \mathrm{CI})$ & $P$ \\
\hline \multicolumn{7}{|l|}{ Sex } \\
\hline Female & 1 & -- & -- & 1 & -- & -- \\
\hline Male & 1.272 & $0.651-2.486$ & 0.482 & 1.756 & $0.627-4.918$ & 0.284 \\
\hline \multicolumn{7}{|l|}{ Age (years) } \\
\hline$<50$ & 1 & -- & -- & 1 & -- & -- \\
\hline$\geq 50$ & 0.328 & $0.152-0.711$ & 0.105 & 0.602 & $0.200-1.817$ & 0.368 \\
\hline \multicolumn{7}{|l|}{ Race } \\
\hline Han & 1 & -- & -- & 1 & -- & -- \\
\hline Zhuang & 0.854 & $0.423-1.721$ & 0.658 & 0.704 & $0.234-2.115$ & 0.532 \\
\hline \multicolumn{7}{|c|}{ Tumor size (cm) } \\
\hline$<8$ & 1 & -- & -- & 1 & -- & -- \\
\hline$\geq 8$ & 5.42 & $2.607-11.27$ & $<0.001$ & 7.428 & $2.529-21.820$ & $<0.001$ \\
\hline \multicolumn{7}{|c|}{ Tumor location } \\
\hline Stomach & 1 & -- & -- & 1 & -- & -- \\
\hline Intestines & 3.710 & $1.716-8.021$ & 0.001 & 1.891 & $0.648-5.518$ & 0.244 \\
\hline Enterocoelia & 0.098 & $0.012-0.788$ & 0.028 & 0.080 & $0.007-0.926$ & 0.043 \\
\hline \multicolumn{7}{|l|}{ Genotyping } \\
\hline KIT exon 11 & 1 & -- & -- & 1 & -- & -- \\
\hline KIT exon 9 & 1.591 & $0.652-3.884$ & 0.308 & 1.080 & $0.308-3.785$ & 0.105 \\
\hline $\begin{array}{l}\text { Wild-type KIT } \\
\text { and PDGFRA }\end{array}$ & 1.591 & $0.413-6.133$ & 0.105 & 1.158 & $0.214-6.267$ & 0.105 \\
\hline Not available & 0.089 & $0.031-0.255$ & $<0.001$ & 0.069 & $0.019-0.255$ & $<0.001$ \\
\hline
\end{tabular}

$1=$ Referent
Table 3. Multivariate Analysis of Cancer-Specific Survival (CSS)

\begin{tabular}{|c|c|c|c|}
\hline & HR & $P$ & $(95 \% \mathrm{CI})$ \\
\hline \multicolumn{4}{|l|}{ Sex } \\
\hline Female & 1 & -- & -- \\
\hline Male & 1.113 & $0.559-2.215$ & 0.761 \\
\hline \multicolumn{4}{|l|}{ Age (years) } \\
\hline$<50$ & 1 & -- & -- \\
\hline$\geq 50$ & 1.763 & $0.897-3.463$ & 0.100 \\
\hline \multicolumn{4}{|l|}{ Race } \\
\hline Han & 1 & -- & -- \\
\hline Zhuang & 0.707 & $0.304-1.640$ & 0.419 \\
\hline \multicolumn{4}{|l|}{ Tumor size $(\mathrm{cm})$} \\
\hline$<8$ & 1 & -- & -- \\
\hline$\geq 8$ & 2.482 & $1.264-4.874$ & 0.008 \\
\hline \multicolumn{4}{|l|}{ Tumor location } \\
\hline Stomach & 1 & -- & -- \\
\hline Intestines & 0.849 & $0.393-1.834$ & 0.676 \\
\hline Enterocoelia & 0.74 & $0.279-1.963$ & 0.545 \\
\hline \multicolumn{4}{|l|}{ Treatment } \\
\hline $\begin{array}{l}\text { No-Neoadjuvant } \\
\text { Imatinib }\end{array}$ & 1 & -- & -- \\
\hline $\begin{array}{l}\text { Neoadjuvant } \\
\text { Imatinib }\end{array}$ & 0.197 & $0.046-0.833$ & 0.027 \\
\hline \multicolumn{4}{|l|}{ Genotyping } \\
\hline KIT exon 11 & 1 & -- & -- \\
\hline KIT exon 9 & 0.789 & $0.151-4.135$ & 0.779 \\
\hline $\begin{array}{l}\text { Wild-type KIT and } \\
\text { PDGFRA }\end{array}$ & 3.198 & $0.664-16.405$ & 0.147 \\
\hline Not available & 2.831 & 1.119-7.162 & 0.028 \\
\hline
\end{tabular}

In order to find out clinical threshold when was the occurrence of resistance, we suspect whether the variation of side effects could be the hints. Widespread inhibition of PDGFR and VEGFR lead to complication of thrombocytopenia and hemorrhage especially among bulky tumor, bleeding occurred rarely but lethal to some circumstance with artery invasion[32]. To reduce this complication, at lease 6 weeks blank period of imatinib should be allowed in pre-operation, and being reevaluated by blood test. Since the vomiting and nausea would be the result of inhibition of Cajal cell [32], the emergence might be an indicator for evaluating drug effect at the beginning. The other major side effect would be edema, though its etiology was mainly due to the long duration of suppressing VEGFR. Periphery or regional edema normally could be tolerated, but mesentery edema would challenge the operation which might induce more fragility of tissues. Evidence showed a paradoxical effect on promoting through inhibiting ABL pathway or eliminating edema via inhibiting Arg pathway [32-34]. No related reported has proved a precise mechanism of edema, we can only suspect the more stronger binding effect that cause edema existed in ABL-imatinib pathway counteracted parts of eliminate edema of VEGFR-imatinib pathway whose downstream site was Arg [34-36]. But when edema disappeared, whether a sign of loss of function in ABL inhibition and association of initiating resistance is 
worthy to be studied. In our study, we suggest neoadjuvant imatinib treatment of surgical resection. The reasons include, 1) downsizing the tumor or controlling the diffusion to create surgical chance; 2 ) delaying the incidence of recurrence; 3) improving prognosis. Some of the treatment ended with resistance to imatinib, so the duration of imatinib was not even. However, researches had controversy on whether one year or three years treatment would gain the most benefits after surgery $[16,17,36]$. The design of study tried to avoid the selection bias, the good respond one of preoperatively administrated imatinib could degrade their disease tier and reach the criteria as some of the direct $\mathrm{R} 0$ section ones. But the patients accepted direct $\mathrm{R} 1$ or $\mathrm{R} 2$ resection reflected the more dangerous and unstable condition when they was on the imatinib treatment. It can not be omitted the patient with organ dysfunction or heavy tumor burden only experienced shorter treatment and survival time.

GIST initiation was typical by receptor mutation of tyrosine kinase [21]which was synergistically facilitated by activation of cytokine signaling of proliferation and angiogenesis [4]. We proposed a series of medical intervention involving the possible biological mechanism in Figure 3. Many growth factor receptors contained tyrosine kinase residues where the functional $\mathrm{SH} 2$ domain dominated the autophosphorylation [8]. Through crossed-linking by the bridge sites, those activation of receptor residues, such as EGFR, VEGFR, PDGFR- $\alpha$ and FGFR, shared the same PI3K/AKT downstream axis diverted signals to proliferation [22, 23]. Indeed we have limited data to estimate which pathways was predominantly activated in tumor progression. Once the phosphorylation completed, a complex inserted to a 'docking site' on juxtamembrane followed by the escalating activation of PI3K. Therefore, inhibition of proliferation via PI3K/AKT would be the crucial overlapped downstream pathway when various blocking of tyrosine kinase receptors were induced [24]. Besides, the recruitment of PI3K passed the apoptotic signal to AKT, but aiming at suppress p53-dependent or -independent apoptosis [24, 25].

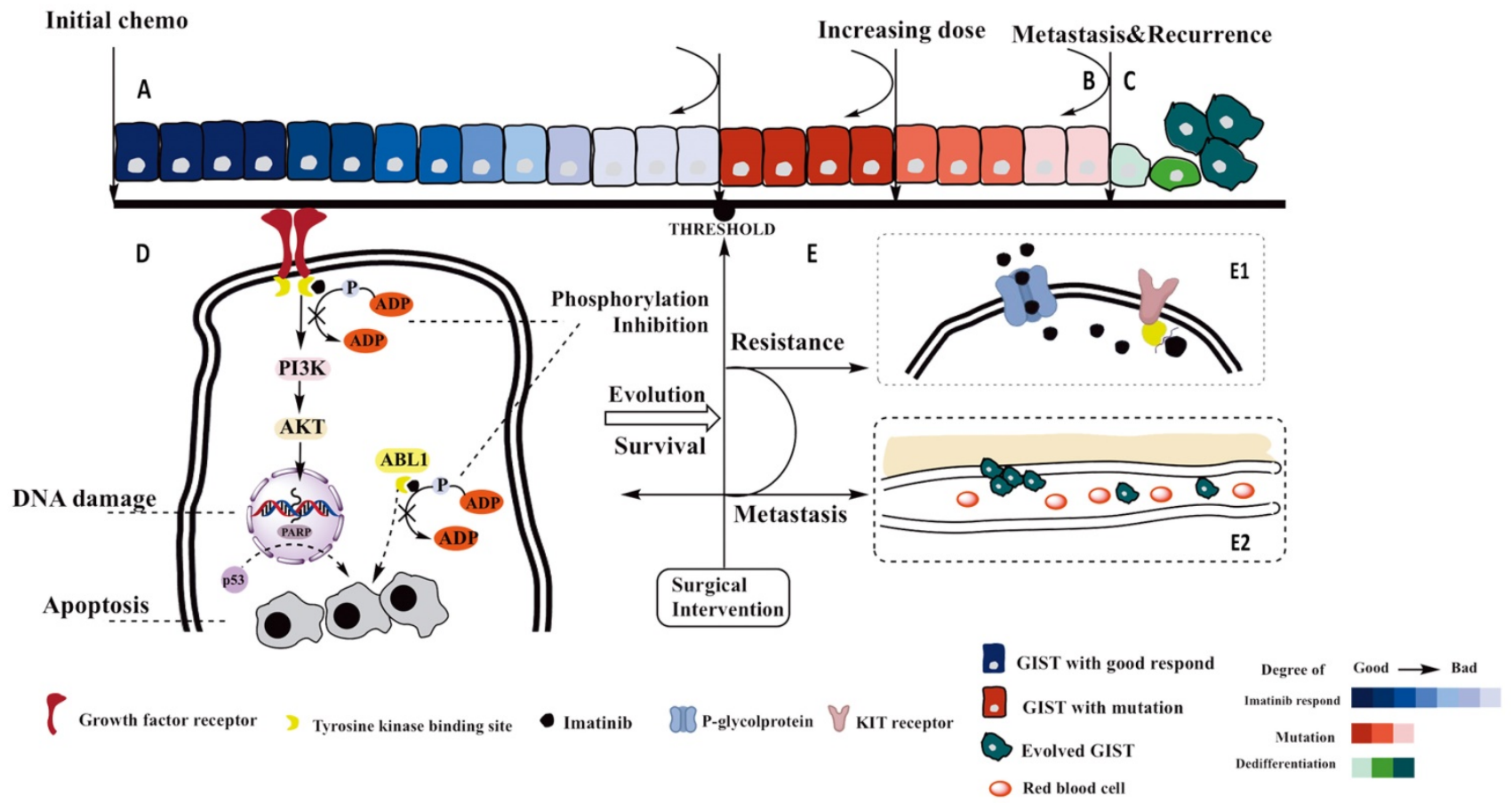

Figure 3. Cell line simulated the putative progression of GIST cell and the possible mechanism of tumor progression. The section A: gradients of blue cell indicated the first medication with imatinib, Dark blue indicated good respond to imatinib whereas the gradually faded blue indicated that decreased effect of imatinib due to the survival GIST potentially evolved to mutation. Correspond to section $\mathbf{D}$, where a possible mechanism was illustrated in how tumor progressed and be inhibited. Section B demonstrated the progression of mutated GIST. Dark red cells represented the Increasing expression level of multidrug resistance protein (MDRI) and process of KIT trans-configuration, the more active P-glycoprotein existed, the less imatinib stayed in GIST cells. At this stage, increasing dose can still maintained sufficient intracellular concentration of imatinib. Section E showed the detailed of mechanism of resistance and metastasis. When KIT-receptors finished configuration mutation, Imatinib failed to bind those receptors, which lead to recovery of tumor progression (Section E1). Section C: If no intervention can conquer mutation, cell would dedifferentiate itself, the lose of cell adhesion made those nidus travelled with circulation, resulting in unpredictable metastasis (Section E2). Between section $\mathbf{A}$ and $\mathbf{B}$, if the surgical intervention would timely conduct at the threshold, we suspected the effect of therapy could be recovered since the evolved cell has been removed. 
As evolution is the nature of all organism, resistance eventually would happen on tumor cells if long exposure of chemo-reagents existed. Once it happened, overlapped standard therapy lead to dramatically decreased efficacy and selectivity of binding sites, resulting in more side effects wherein the hemorrhagic tendency ranked the most risk to surgeon. There is no standard criteria can surgeon assist for estimating early on-set of resistance. This threshold of when is the timing of surgical intervention challenged clinical practice. In our hypothesis, removal of resistant lesion postponed the metastasis and resistance, as well as guarantee in vivo efficacy of medication. Specifically to detail the resistance, which included the over-activation of P-glucose protein and structurally transformation of 'docking site' [26-28]. At this level, solution could be replaced by second-line regents, or dose escalation of imatinib up to $800 \mathrm{mg}$ [28]. Escalation of imatinib competed the efflux of drug molecule which depended on the over-contaction between molecule and 'docking sites', compensating the insufficient block [29]. But dose of $800 \mathrm{mg}$ imatinib to primary GIST patients needs careful evaluation because it would gain more toxicity rather than better outcomes $[30,31]$. Additionally, genotyping is another factor to predict where the respond level of imatinib treatment deviation would reach.

\section{Conclusion}

Although popularity of GIST has been raised in coming decades, still, many of GIST patients were vaguely classified as gastric cancer. Additionally, lack of precise immunohistochemical and pathological analysis downsizes sample scale. Some of GIST patients accompanied with comorbidity such as diabetes, hyperlipemia and pulmonary hypertension was coincidently regression due to medication with imatinib. What urgently to be promoted roots to the optimal medical combination for GIST.

\section{Supplementary Material}

Supplementary tables.

http://www.jcancer.org/v09p3850s1.pdf

\section{Acknowledgments}

We acknowledge financial support from the National Science Foundation for Young Scholars of China (No. 81502120 and 81560454) and Guangxi Medical University Training Program for Distinguished Young Scholars. We gratitude the technology support from Prof. Wei-Zhong Tang in helping us with software problem.

\section{Competing Interests}

The authors have declared that no competing interest exists.

\section{References}

1. Longo DL. Imatinib Changed Everything. N Engl J Med 2017; 376: 982-983.

2. Freireich EJ, Wiernik PH, Steensma DP. The leukemias: a half-century of discovery. J Clin Oncol 2014; 32: 3463-3469.

3. Mandavilli A. Leukaemia. Nature 2013; 498: S1.

4. Fischer PM. Approved and Experimental Small-Molecule Oncology Kinase Inhibitor Drugs: A Mid-2016 Overview. Med Res Rev 2017; 37: 314-367.

5. Hochhaus A, Larson RA, Guilhot F et al. Long-Term Outcomes of Imatinib Treatment for Chronic Myeloid Leukemia. N Engl J Med 2017; 376: 917-927.

6. Cioffi A, Maki RG. GI Stromal Tumors: 15 Years of Lessons From a Rare Cancer. J Clin Oncol 2015; 33: 1849-1854.

7. Levitzki A, Mishani E. Tyrphostins and other tyrosine kinase inhibitors. Annu Rev Biochem 2006; 75: 93-109.

8. Corless CL, Barnett CM, Heinrich MC. Gastrointestinal stromal tumours: origin and molecular oncology. Nat Rev Cancer 2011; 11: 865-878.

9. Hornick JL, Fletcher CD. The role of KIT in the management of patients with gastrointestinal stromal tumors. Hum Pathol 2007; 38: 679-687.

10. Ma GL, Murphy JD, Martinez ME, Sicklick JK. Epidemiology of gastrointestinal stromal tumors in the era of histology codes: results of a population-based study. Cancer Epidemiol Biomarkers Prev 2015; 24: 298-302.

11. Coe TM, Fero KE, Fanta PT et al. Population-Based Epidemiology and Mortality of Small Malignant Gastrointestinal Stromal Tumors in the USA. J Gastrointest Surg 2016; 20: 1132-1140.

12. Mussi C, Ronellenfitsch U, Jakob J et al. Post-imatinib surgery in advanced/metastatic GIST: is it worthwhile in all patients? Ann Oncol 2010; 21: 403-408.

13. Wilkinson MJ, Fitzgerald JE, Strauss DC et al. Surgical treatment of gastrointestinal stromal tumour of the rectum in the era of imatinib. Br J Surg 2015; 102: 965-971.

14. Fero KE, Coe TM, Fanta PT et al. Surgical Management of Adolescents and Young Adults With Gastrointestinal Stromal Tumors: A US Population-Based Analysis. JAMA Surg 2017; 152: 443-451.

15. Lin JX, Chen QF, Zheng CH et al. Is 3-years duration of adjuvant imatinib mesylate treatment sufficient for patients with high-risk gastrointestinal stromal tumor? A study based on long-term follow-up. J Cancer Res Clin Oncol 2017; 143: 727-734.

16. Joensuu H, Eriksson M, Sundby Hall K et al. One vs three years of adjuvant imatinib for operable gastrointestinal stromal tumor: a randomized trial. Jama 2012; 307: 1265-1272.

17. Yoo C, Ryu MH, Kang BW et al. Cross-sectional study of imatinib plasma trough levels in patients with advanced gastrointestinal stromal tumors: impact of gastrointestinal resection on exposure to imatinib. J Clin Oncol 2010; 28: 1554-1559.

18. Boikos SA, Pappo AS, Killian JK et al. Molecular Subtypes of KIT/PDGFRA Wild-Type Gastrointestinal Stromal Tumors: A Report From the National Institutes of Health Gastrointestinal Stromal Tumor Clinic. JAMA Oncol 2016; 2: 922-928.

19. Poveda A, Garcia Del Muro X, Lopez-Guerrero JA et al. GEIS guidelines for gastrointestinal sarcomas (GIST). Cancer Treat Rev 2017; 55: 107-119.

20. Joensuu H. Risk stratification of patients diagnosed with gastrointestinal stromal tumor. Hum Pathol 2008; 39: 1411-1419.

21. Krause DS, Van Etten RA. Tyrosine kinases as targets for cancer therapy. N Engl J Med 2005; 353: 172-187.

22. Bauer TM, Patel MR, Infante JR. Targeting PI3 kinase in cancer. Pharmacology \& therapeutics 2015; 146: 53-60.

23. Lee MS, Jeong MH, Lee $\mathrm{HW}$ et al. PI3K/AKT activation induces PTEN ubiquitination and destabilization accelerating tumourigenesis. Nat Commun 2015; 6: 7769.

24. LoRusso PM. Inhibition of the PI3K/AKT/mTOR Pathway in Solid Tumors. Journal of clinical oncology : official journal of the American Society of Clinical Oncology 2016; 34: 3803-15

25. Tian X, Huang $\mathrm{B}$, Zhang XP et al. Modeling the response of a tumor-suppressive network to mitogenic and oncogenic signals. Proc Natl Acad Sci U S A 2017; 114: 5337-5342.

26. Nemethova V, Razga F. Overexpression of $\mathrm{ABCB} 1$ as prediction marker for CML: How close we are to translation into clinics? Leukemia 2017; 31: 266-267.

27. Weisberg E, Griffin JD. Resistance to imatinib (Glivec): update on clinical mechanisms. Drug Resist Updat 2003; 6: 231-238.

28. Patel S. Managing progressive disease in patients with GIST: factors to consider besides acquired secondary tyrosine kinase inhibitor resistance. Cancer Treat Rev 2012; 38: 467-472.

29. Kosztyu P, Bukvova R, Dolezel P et al. Resistance to daunorubicin, imatinib, or nilotinib depends on expression levels of ABCB1 and ABCG2 in human leukemia cells. Chem Biol Interact 2014; 219: 203-210.

30. Verweij J, Casali PG, Zalcberg J et al. Progression-free survival in gastrointestinal stromal tumours with high-dose imatinib: randomised trial. Lancet 2004; 364: 1127-1134. 
31. Casali PG, Zalcberg J, Le Cesne A et al. Ten-Year Progression-Free and Overall Survival in Patients With Unresectable or Metastatic GI Stromal Tumors: Long-Term Analysis of the European Organisation for Research and Treatment of Cancer, Italian Sarcoma Group, and Australasian Gastrointestinal Trials Group Intergroup Phase III Randomized Trial on Imatinib at Two Dose Levels. J Clin Oncol 2017; 35: 1713-1720.

32. Joensuu H, Trent JC, Reichardt P. Practical management of tyrosine kinase inhibitor-associated side effects in GIST. Cancer treatment reviews 2011; 37: 75-88.

33. Aman J, van Bezu J, Damanafshan A et al. Effective treatment of edema and endothelial barrier dysfunction with imatinib. Circulation 2012; 126: 2728-38.

34. Aman J, Peters MJ, Weenink $C$ et al. Reversal of vascular leak with imatinib. American journal of respiratory and critical care medicine 2013; 188: 1171-3.

35. Penn MS, Kamath M. Novel mechanisms for maintaining endothelial barrier function in sepsis. Circulation 2012; 126: 2677-9.

36. Joensuu H, Eriksson M, Sundby Hall K et al. Adjuvant Imatinib for High-Risk GI Stromal Tumor: Analysis of a Randomized Trial. J Clin Oncol 2016; 34: 244-250. 